\title{
COVID 19 and Psychological Impact across Lifespan
}

\author{
Nadigapu Dayanand Sanjay Kumar \\ *Corresponding author: \\ Dr. N.D. Sanjaykumar, Senior Resident, Department of Psychiatry, Prathima Institute of Medical Sciences, Karimnagar, \\ Telangana, India \\ drsk2010@gmail.com \\ DOI : 10.47799/pimr.0802.03
}

Introduction

COVID-19 the present pandemic like all pandemics puts a strain on both the individual and the community. Human beings are social animals; they need interactions with other human beings in order to maintain good mental health, the present pandemic put a restriction on interactions between people in the form of social distancing, wearing of masks, use of sanitizers and soap and water, which are essential to control the spread of the corona virus but the flip side, it has reduced the family gatherings, going out for restaurants, factors to a great extent that will have impact on psychological health.

Health infrastructure and health services across the globe are under enormous pressure and face unprecedented challenges. Medical professionals, health care workers, scientists and other front line workers are on the forefront on one hand they are providing health care to the Covid 19 affected patients and the other hand, building systemic responses both by dissemination of adequate public health approaches, and in parallel, genetic mapping, drug and vaccine development. Every day millions are being infected and thousands have died. This pandemic has put all aspects of normal life on hold. In this scenario mental health care takes a back seat to life or death decisions made by the exhausted health care workers. ${ }^{1}$

\section{COVID-19 has a hidden pandemic of emotional distress}

The psychological effects of the pandemic are best understood in terms of the psychiatric and psychological problems that were present before the pandemic and the pathoplastic affects of the pandemic on the problems; the responses to social isolation and lockdown; the psychological responses to the diagnosis; how the public responds to those with symptoms of Covid 19 infection as well as after the infection is confirmed.

In India, the first and foremost response to the pandemic was fear and sense of imminent danger. Fears are based on information or misinformation circulating in the media and in particular social media. Each individual responds differently to the flooding of information from various sources. This can lead to those who are the "worried well", those who develop distressful psychological symptoms and maladaptive

coping with stress, and those who develop a mental disorder. The fear of contracting the illness is also frequent and range from misinterpreting every fever or cough as a Covid 19 infection, wanting a test done for reassurance even though there are strict guidelines for testing, to hoarding medications despite there not being indications for their generalised use. Also the fears about whether the precautionary measures are adequate or not, doubts about the protection from infections can also add to constant feelings of distress in people. ${ }^{2}$

Apart from maintaining hygiene, the mechanism stopping the spread is social distancing (also known as physical distancing). It is the very antithesis of all that is considered therapeutic in psychological health. Human brains for millennia are hardwired for social contact; enforced isolation deprives humans of social contact. In human primates, the need for social behaviour is as keenly experienced as that of hunger. Social distancing takes away warmth, support and feeling of belongingness when you need it the most.

In this scenario, mental or emotional distress linked to social losses has to be addressed efficiently. With the economic slowdown and layoffs from jobs there are already reports of businessmen committing suicides and this may increase further if not addressed the issue proactively. There will be various anxiety issues, where any small bodily ache or fever or cold will be viewed as COVID -19. This may lead to excessive thinking in people thereby leading to loss of control and a feeling of hopelessness. Similar scenarios were witnessed during Spanish Flu, the pandemic of 1918 and the Great Depression in 1930. On the other extreme there may be a population who are also completely unworried or who do not follow the advisory precautions. This attitude can also lead to an endangerment to self and others and bring a feeling of helplessness in the rest.

The most distressing aspect of mental health care is that it has not kept pace with the other health care in terms of accessibility, in part due to a lack of parity in insurance coverage for mental health. In India and many nations 'specialist' outpatient services are largely the promise of private practitioners and thus affordable for only a few. Public mental health services are plagued with shortage of staff and overburdened with patients. The people with severe mental 
disorders having an acute episode from the low socio economic status are being particularly affected. They are being managed and monitored at home, in the background of food scarcity, loss of employment and no regular income.

Mental health care cannot be completely given by tele psychiatry or by means of computers, it puts burden on the patients to have means to consult by computer or phone. Alienation generated by the absence of social ties and exacerbations of conflicts experienced within families can lead to domestic crisis.

For majority of people who are caught in the web of economic marginalization, social disadvantage, inequity, gender disparity and powerlessness, the human experience of the pandemic and related social losses could further push people into hopelessness. Globally, health, social and economic inequities stand in the open today, more than before.

With the striking of Covid 19 at this juncture, the already deepening crisis of rising economic inequalities and homelessness in much of the world has made the situation open to all to witness. Mandatory 'stay at home' policies have been a cruel reminder that people who are homeless and or who have a minimal space to stay constantly. Lock down in many parts of the world has exposed other vulnerable groups and communities to further risk. Migrant workers were the other set who were affected badly by the lockdown. In India, much of the labour force is actually migrants from other states.

Women have become the most vulnerable group with an increase in domestic violence incidents being reported world over. Casteism, classism and cruel antisocial behaviours are often both increased and justified in such times, resulting in further alienation and herd mentality and group identity gets the better of reason, humanity and social connectedness. Meanwhile, the wealthy are able to live in capacious homes and the underpaid and at risk essential workers who maintain transportation networks, food supply chains and the all important health care that sustains society's vital infrastructure find it difficult to find their space. The burdens of inequality have increased exponentially.

The implications of Covid- 19 on children, adolescents and families

The Covid-S 19 pandemic has the potential to impose far reaching and long term negative consequences on children around the world. According to an estimate there is going to be around 10-40 million deaths and some others due to suicide leaving many children parentless. During covid-19, if the mother contracts the illness, then she has to stay in isolation leading to extreme anxiety in children. The idea of staying at home imposes curb on most physical activities for the children cutting down on their play time and peer relationships. With lack of social stimulation and restricted area and parents working from home, most children will depend on internet and digital media for keeping themselves entertained. The increased screen time for children is studied to have long term negative consequences in their brain development and the constant exposure to radiation and lacking physical activity can together lead to many behavioural problems such as clinginess, distraction, irritability, lethargy, decline in social skills, boredom and common psychological problems such as anxiety and depression in general. The children of families who have been affected with Covid may experience trauma due to isolation, grief due to loss of loved ones and fear of being falling sick or loneliness.

In April 2020, Spain had the world's second highest number of covid 19 deaths. In 7 million youngsters who were indoors due to lockdown struggled with increased levels of stress and anxiety, insomnia, chest and stomach pain and weight gain. A study by the Kaiser Family foundation in USA predicted that adolescents at risk for depression or suicidal ideation were at high risk for manifestation of depression, anxiety, distress and low self esteem. ${ }^{3}$

\section{Adjustment Disorders}

The Oxford study findings showed that different age groups show variation in the expressions of anxiety and stress reactions. Younger children worry about catching the infection and fear of infecting others and anxiety about missing school. Older children worry more about their families, friends and relatives than about themselves. Interestingly, anxiety levels among parents were much higher than compared to their children. Social isolation increases anxiety, depression, phobias, stress and associated negative feelings. With an increased focus on washing of hands and cleaning protocol for people getting in contact with others, there has been an increase in the obsessive compulsive behaviours and the constant fear of contraction of the illness may for a few instil paranoia.

Scenario of People with Mental Illness during Covid-19

Pre-existing mental health conditions such as ADHD, mood and anxiety disorders, Autism spectrum disorders, Obsessive compulsive disorders, post traumatic stress disorder and abuse of alcohol and psychoactive substances can worsen further.

\section{Coping in the Covid times}

The restrictions of the pandemic cannot be avoided and need to be followed for the larger good and hence it is important for us to understand how to nurture our mental health within the context of Covid-19. The first step to coping would be to understand, mental health is equally important as physical health and the same care you give to your body is needed for your mind too. As we take good care of our physical 
health by eating well or maintaining hygiene, the need to keep our social relationships active is required for our emotional well being. Social interactions have to be emphasized either face to face (with the necessary precautions) or virtual.

Our lifestyle has an impact on our well being. Sleep is an important determinant of mental health. A good restful sleep of 8 hours will reduce the stress levels considerably. A fixed time to sleep and wake up is important. Your routine should include a regular exercise regime which includes both physical and mental activities. Meditation or breathing exercises are proven to be effective in maintaining good mental health. You should also follow a routine to keep yourself active. A balanced nutrition is inevitable for good health as a whole and that includes mental health.

With the onset of the pandemic uncertainty has become prominent; uncertainty about jobs, migration and any sort of plan changes. This has created a lot of anxiety in people about the security about their future or about getting infected with the virus and also with lot of restrictions; the mobility has come down. This has in turn led to a lot of stress in the public. Practise of mindfulness will largely be able to deal with the stress related to worrying about future and overthinking. This helps one in shifting the focus from the ruminations of the past events or future worries to the present moment giving oneself control over their own thoughts.

As this has become a time where you spend more time with your family, this may also give you an opportunity to spend quality time with your family and developing understanding between each other. On the contrary, there has been an increase in the number of incidents of violence and conflicts in the family as with constant presence and lack of space causing adjustment problems in the family. The role of mental health professionals is significant in such scenarios and the differences may be sorted with professional interventions.

\section{Conclusion}

Humanity has faced many greater crises in the past both manmade and natural and every time we have bounced back. The strength of the human spirit is enormous and we will emerge victorious from this pandemic too. This is possible only when we are able to let go of our efforts to know everything and to accept the constancy called change and learn to take one step at a time and taking all the precautions advised by the government and the advisory board. In this hour of crisis being united in our fight against Covid 19 will take us to the point of victory with co operation, coordination and pooling up of all the resources at our disposal.

\section{REFERENCES}

1. Gopikumar V, Padgett DK. Sarin A, Mezzina R, Willford A, Jain S. Mental Health and Corona Virus: A Global Perspective. World Social Psychiatry 2020; 2:88-93.

2. Mental health in the times of COVID 19 pandemicGuidance for general medical and specialized Mental Health Care Settings, NIMHANS

3. Chottera C S, Doughlas D- Bright AM, Sedky K, Gogineni RR, Rostain A L. COVID- 19, Social Distancing: Mental Health Implications for Children, Adolescents, and Families- Pediatric and Psychiatric Perspectives. World Social Psychiatry 2020; 2: 159-62.

How to cite this article : Sanjay kumar ND. COVID 19 and Psychological Impact across Lifespan. Perspectives in Medical Research 2020; 8 (2):4-6

DOI : 10.47799/pimr.0802.03

Sources of Support: Nil,Conflict of interest:None declared 